\title{
"Soy feminista pero...": afectos, humor e identificación en The Guilty Feminist
}

\section{"I am a feminist but...": affects, humor and identification in The Guilty Feminist}

\author{
Mariela Solana \\ mariela.solana@gmail.com \\ Universidad Nacional Arturo Jauretche - Universidad \\ de Buenos Aires - Consejo Nacional de Investigaciones \\ Científicas y Técnicas, Argentina
}

Recepción: 30 Marzo 2019

Aprobación: 27 Abril 2020

Publicación: 01 Marzo 2021

Cita sugerida: Solana, M. (2021). "Soy feminista pero...": afectos, humor e identificación en The Guilty Feminist. Descentrada, 5(1), e135. https://doi.org/10.24215/25457284e135

\begin{abstract}
Resumen: El objetivo de este artículo es analizar los afectos que circulan en el podcast cómico The Guilty Feminist, con el fin de determinar cuál es la clase de "nosotras-feministas" que se gesta en el encuentro entre el chiste de las comediantes y la respuesta de la audiencia. El podcast es parte de una nueva generación de humor feminista cuyo objeto de burla es el modo en que somos, y creemos que debemos ser, feministas. El artículo explora aquellas situaciones cómicas que generan una respuesta favorable en la audiencia así como la irrupción, por momentos, de silencios incómodos y gestos de reprobación. La meta es comprender el proceso de identificación que estas situaciones habilitan y que producen una comunidad feminista basada en sentimientos como la culpa, la vergüenza, la incomodidad y la frustración.
\end{abstract}

Palabras clave: Humor feminista, Culpa, Comunidad afectiva, Cuarta ola feminista.

\begin{abstract}
The goal of this article is to examine the affects that circulate in the comedy podcast The Guilty Feminist in order to determine what sort of feminist community emerges in the encounter between the joke of the comedians and the response of the audience. This podcast is part of a new generation of feminist humor that makes fun of the way we are, and think we ought to be, feminists. The article explores those funny situations that generate a favorable response in the audience and the irruption, at times, of awkward silences and gestures of disapproval. The goal is to understand the process of identification that these situations create and that produce a feminist community based on feelings such as guilt, shame, awkwardness and frustration.
\end{abstract}

Keywords: Feminist humor, Guilt, Affective community, Fourth-wave feminism.

Nuestra risa es siempre la risa de un grupo. Henri Bergson, La risa. Ensayo sobre la significación de lo cómico.

\section{INTRODUCCIÓN}

"Soy feminista pero, algunos días, mi vida ni siquiera pasa el test de Bechdel" (Frances-White, TGF, 15/12/2015). "Soy feminista pero, una vez, le dije a un hombre '¿qué tengo que cambiar de mí para que me ames?'” (Hagen, TGF, 15/12/2015). Estas son algunas de las frases con las que se inicia el primer episodio 
de The Guilty Feminist (TGF), un podcast cómico conducido actualmente por Deborah Frances-White y grabado ante una audiencia en vivo. ${ }^{1}$ El tema del podcast, como se explicita en cada episodio, gira en torno a las inseguridades, hipocresías y miedos que experimentamos como feministas en el siglo XXI. TGF es parte de una nueva generación de humor feminista, cuyo objeto de burla no es tanto el sistema heteropatriarcal sino el modo en que somos, y creemos que debemos ser, feministas. Este humor metafeminista, como me gustaría denominarlo, pone en primer plano que los estereotipos, prejuicios e ideales a seguir no son solo imposiciones externas sino internas, es decir, que emanan desde adentro mismo del movimiento feminista y desde las propias entrañas de quienes nos consideramos feministas. A pesar de que el feminismo está atravesando un momento de gran exposición mediática y validación pública, seguimos habitando un mundo fuertemente marcado por la violencia y los prejuicios sexistas. TGF permite comprender que esta violencia y estos prejuicios son males que no solo padecemos, sino que también reproducimos en nuestras prácticas cotidianas. Ser feminista, según esta perspectiva, no significa liberarse de forma absoluta de los mandatos patriarcales, sino aprender a navegar las propias contradicciones e incongruencias. El podcast apuesta a que la mejor forma de hacer esto es con una buena dosis de humor e ironía.

El objetivo de este artículo es analizar los afectos que circulan en TGF -a veces, alegres, por momentos, tristes- para determinar cuál es la clase de "nosotras-feministas" que se produce en el encuentro entre el chiste de las comediantes y la respuesta de la audiencia. Como señalan Lauren Berlant y Sianne Ngai (2017, p. 243): "La comedia nos ayuda a testear o entender qué significa decir 'nosotrxs'. Siempre cruzando líneas, nos ayuda a entender qué líneas deseamos o podemos soportar". ${ }^{2}$ Siguiendo esta idea, este trabajo explora cómo, en TGF, se va gestando un tipo particular de comunidad feminista a partir del reconocimiento de que hay ciertas concesiones que son compatibles con una vida feminista y otras que, en cambio, resultan más difíciles de armonizar. Para esto, se examina el papel de la risa, así como la irrupción de silencios incómodos y gestos de reprobación, como parte de un proceso de identificación entre la audiencia y las cómicas. Algunos estudios humorísticos sostienen que el éxito de la comedia depende, justamente, de que se logre este proceso de identificación (Krefting, 2014; Merrill, 1988). Según la teórica del humor Rebecca Krefting, por ejemplo, "si bien la identificación no es el único prerrequisito para disfrutar un chiste, ayuda a que la comedia sea exitosa. En términos generales, esto se traduce en 'entiendo lo que decís y mi risa es un signo de mi aceptación o afirmación'." (2014, p. 6).

Uno de los puntos que quisiera demostrar en estas páginas es que el nosotras-feministas que se gesta en TGF puede ser entendido como una comunidad afectiva. Esto es así, ya que el elemento aglutinante -aquello que produce sentimientos de pertenencia- no es tanto un ideal compartido sino, más bien, sentimientos en común: culpa, vergüenza, incomodidad y frustración. Una comunidad afectiva "puede ser caracterizada como una sensualidad compartida que evoca un sentido implícito de comunidad e inmediatez" (Zink, 2019, p. 289). Se trata de un colectivo cuyo lazo -efímero y contingente- depende de emociones, sentimientos y sensaciones que encuentran eco, resuenan, se tensan, se amplifican (o disminuyen) y, en general, se modulan en el encuentro entre los cuerpos del colectivo. Lo que desarrollaré en breve es que, lejos de sostenerse en una narrativa heroica de empoderamiento y resistencia, el nosotras-feminista que aparece en TGF se basa en el reconocimiento y aceptación de la propia imperfección y de las múltiples contradicciones que horadan las vidas feministas. Si, como afirma Bergson (1962, p. 14), la risa es siempre la risa de un grupo, lo que este trabajo intenta determinar es qué tipo de grupo construye un podcast como TGF. ¿Qué tipo de nosotrasfeminista emerge cuando, en lugar de negarlas, nos burlamos de nuestras inseguridades, culpas e hipocresías?

En segundo lugar, este artículo argumenta que esta nueva forma de hacer comedia permite complejizar algunos de los postulados de los estudios feministas clásicos sobre el humor. Según estos estudios, en oposición al humor machista, la comedia feminista no desprecia a las mujeres, sino que las empodera (Kaufman, 1980; Merrill 1988). TGF, sin embargo, pone en jaque esta división: la risa no surge ni de la denigración de las mujeres ni de subrayar sus fortalezas. Más bien, lo que se nos presenta es una burla no-despreciativa de nuestro 
modo imperfecto de ser y creer que debemos ser feministas; una burla que nace menos de la resistencia que de la exhibición de una vulnerabilidad en común.

Para alcanzar los fines propuestos, este artículo está dividido en cuatro partes. En la primera, se pasa revista a diversas explicaciones de por qué el humor se ha constituido, históricamente, como un dominio masculino. En la segunda, se hace una breve reseña de la relación entre feminismo y comedia, así como de las diferencias entre el humor machista y el feminista. En la tercera, se analiza qué tipo de situaciones cómicas generan mejores respuestas en la audiencia de TGF y qué chistes, en cambio, producen rechazo o reacciones mixtas. Finalmente, en el cuarto apartado, se sintetiza cómo es el tipo de comunidad que se gesta en el podcast y se indaga si TGF puede ser considerado un arquetipo de la clase de humor que la cuarta ola feminista necesita.

\section{2. ¿SON LAS MUJERES GRACIOSAS?}

Como afirman varios estudios anglosajones sobre el humor, ${ }^{3}$ la comedia es y ha sido, históricamente, un espacio masculino (Kaufman, 1980; Merrill; 1988; Krefting, 2014; Kein, 2015; Fuchs Abrams, 2017). ¿Cuál es el motivo? Según una nota polémica escrita por el periodista Christopher Hitchens para la revista Vanity Fair, en 2007, la respuesta es simple: las mujeres no son graciosas. La nota se titula, justamente, “¿Por qué las mujeres no son graciosas?" [“Why Women Aren't Funny?"] e intenta dar cuenta de una división sexual de tareas de seducción: las mujeres impresionan al sexo opuesto con su belleza y los varones con su sentido del humor. Hitchens cita un estudio empírico de la Escuela de Medicina de Stanford, cuyo resultado es que las mujeres son más lentas para entender tiras cómicas y más rápidas para identificar lo que consideran poco divertido. Además, el autor se hace eco de una idea bastante recurrente en estudios sobre género y humor: el humor es agresivo y la agresión es mejor expresada por los varones.

Ahora bien, Hitchens considera que la verdadera clave para entender la falta de humor femenino se encuentra en la biología. Al parecer, las mujeres no son graciosas porque no tienen tiempo para perder con frivolidades y tonterías. Ellas tienen algo mucho más importante que hacer: ser madres. La reproducción, para las mujeres, "si no es la única cosa, es lo más importante... [y] les infunde un tipo de seriedad y solemnidad que los varones solo pueden mirar absortos" (Hitchens, 2007). Haciendo uso de la ironía que, como varón, supone le pertenece, el periodista sostiene que la placenta está conformada por células cerebrales que, durante el embarazo, migran al sur llevándose consigo el sentido del humor. El punto es que, a veces, el humor nunca regresa: “Hay algo con menos sentido del humor que una madre hablando de su nuevx hijx?” (Hitchens, 2007). La posibilidad de parir no solo inocula a las mujeres contra la ridiculez y trivialidad propia de la comedia, sino que además obliga a los varones a ser graciosos para burlarse de la autoridad femenina. Los varones están "impresionados, para no decir aterrorizados, por la habilidad de las mujeres de producir bebés” (Hitchens, 2007) y su destreza en el humor sería su forma de admitir que ellas son las verdaderas jefas. El texto de Hitchens, así, es una muestra de cómo el patriarcado reacciona ante una forma imaginada de poder femenino, reforzando estereotipos biologicistas y negando su capacidad cómica.

Una manera más sofisticada de analizar la exclusión de las mujeres de la esfera humorística, la ofrece Sabrina Fuchs Abrams en el libro Transgressive Humor of American Women Writers (2017). En la introducción, la autora explica que hay tres grandes modelos teóricos sobre el humor -la teoría de la descarga, la teoría de la superioridad y la teoría de la incongruencia- y afirma que es posible recurrir a ellos para entender la postergación de las mujeres de la comedia. Recordemos que la teoría de la descarga -deudora de las reflexiones de Sigmund Freud en El chiste y sus relaciones con el inconsciente, publicado en 1905- asume que al reírnos descargamos energía psíquica y aliviamos tensiones. $\mathrm{Al}$ igual que los sueños, la risa es una forma de liberar pensamientos y sentimientos obscenos que tenemos de modo inconsciente. La teoría de la superioridad, que suele ser adjudicada a Thomas Hobbes en el Leviathan (1998), asume la mentada agresividad de la comedia ya que considera que la risa devalúa el objeto de burla. Finalmente, la teoría de la incongruencia sostiene que 
la risa emerge cuando algo viola nuestras expectativas. Se trata de una perspectiva cognitiva del humor porque asume la capacidad de la mente de ordenar los objetos en categorías familiares.

Según Fuchs Abrams, una podría adoptar cualquiera de estas teorías y negarles capacidad cómica a las mujeres: "la visión tradicional de la mujer como careciendo de tendencias agresivas, sexuales o intelectuales pone en cuestión su habilidad de expresar o incluso de entender el humor" (2017, p. 3). Sin embargo, la autora considera que también hay elementos en estas concepciones clásicas que permiten comprender la comedia realizada por mujeres. La noción que le resulta particularmente útil es la del chiste como una máscara que la autora identifica en la obra de Freud. Según esta perspectiva, el chiste, en ocasiones, tiene potencialidad crítica ya que permite camuflar las hostilidades prohibidas de los grupos postergados. Si bien para Freud, el chiste suele ser utilizado por aquellos en posición de superioridad para reírse de los más débiles, existen posibilidades subversivas cuando son las personas subordinadas quienes hacen uso del humor. Fuchs Abrams acuerda y añade que enmascarar la crítica social bajo la forma del chiste es el modo en que las mujeres comediantes logran transgredir las normas patriarcales.

Finalmente, también existen explicaciones que ponen el foco en el mercado y en los circuitos culturales humorísticos. En el libro All Joking Aside: American Humor and its Discontents (2014), Rebecca Krefting afirma que la pregunta relevante no es por qué las mujeres son menos graciosas que los varones sino cuál es la economía del humor que sustenta esa idea. Si bien Krefting menciona otras explicaciones comunes sobre por qué la comedia suele ser un dominio masculino - por ejemplo, la ya mencionada idea de que hay cierta agresividad intrínseca a la comedia que suele ser censurada en mujeres, o las dificultades que tienen las mujeres para llamar la atención o permitirse hacer el ridículo en público- su tesis principal es que hay que examinar el modo en que la comedia circula socialmente. Para esto, es preciso explorar el complejo entramado de producción, intercambio y consumo de humor: quiénes deciden a quién contratar y a quién no, con qué criterio, qué comedia es publicitada, cuáles son las expectativas de la audiencia y cómo se logra el difícil pero medular proceso de identificación entre audiencia y comediante. Esta forma de pensar al humor y de intentar entender por qué las mujeres han encontrado obstáculos para acceder a ese espacio está en sintonía con las teorías sociales de la risa, como las de Bergson. Según este pensador, la risa

Por muy espontánea que se la crea, siempre oculta un prejuicio de asociación y hasta de complicidad con otros rientes efectivos o imaginarios. ¿No se ha dicho muchas veces que en un teatro es más frecuente la risa del espectador cuando más llena está la sala? ¿No se ha hecho notar reiteradamente que muchos efectos cómicos son intraducibles a otro idioma cuando se refieren a costumbres y a ideas de una sociedad particular? (1962, pp. 14-15).

Ese nosotrxs que se ríe lo hace porque el chiste descansa sobre normas y valores compartidos, incluso cuando los ponga en jaque o se burle de ellos. Son estas creencias compartidas las que funcionan como condición de posibilidad para la identificación entre audiencia y comediante. La comedia exitosa, así entendida, no solo se basa en experiencias comunes, sino que construye comunidad: la comunidad de quienes nos reímos, de quienes entendemos el chiste, de quienes podemos ponernos en los zapatos de lxs comediantes.

Asumiendo estas ideas, Krefting señala que la identificación con comediantes mujeres es menos frecuente:

Las audiencias mainstream -aquí definidas como aquellas con poder para comprar una entrada, sin tener en cuenta su orientación sexual, raza/etnicidad, nación de origen o religión - tienden a afirmar la perspectiva e identificarse (léase: invertir y apoyar económicamente; reír o al menos responder favorablemente) con el cómico varón (2017, pp.107-108).

¿Por qué resulta más sencillo, para las audiencias, identificarse con el comediante varón? Por un lado, la autora señala que lo que suele suceder es que la audiencia considera al humor masculino como humor general y al humor hecho por mujeres como algo específico. Mientras la perspectiva del cómico varón es considerada default, se cree, en cambio, que las comediantes mujeres abordarán temas especiales como las relaciones amorosas, los zapatos, la dieta, etc. -y, solo para dar un ejemplo, Krefting recuerda que el cómico Robert Kelly dijo que una colega mujer era graciosa solo cuando no hablaba de ser mujer- (2017, p. 113). Por otro lado, la autora afirma que es más fácil para la audiencia identificarse con un sujeto privilegiado - 
en este caso, con el comediante masculino (heterosexual, blanco y cis) - que con una posición minoritaria. De acuerdo con Krefting,

la identificación y recepción está preparada desde el inicio para que consumamos las cosas que queremos ser, las cosas que significan poder. El consumo favorece lo que es 'como' nosotrxs y lo que es ideal, lo que tiene el mayor caché cultural y político (2017, p. 119).

Por último, dado que muchas comediantes mujeres dedican parte de su acto a burlarse y, por ende, a sacar a la luz el machismo y la misoginia existentes, esto obliga a la audiencia a tener que asumir una posición crítica y no todxs están dispuestxs o incentivadxs para hacerlo, especialmente quienes están en una posición de privilegio.

En este apartado, se presentaron varias teorías sobre el humor que intentan explicar la menor presencia de comediantes mujeres en los espacios humorísticos. Ya sea que una adopte una posición biologicista, psicoanalítica o culturalista, podríamos afirmar que hay dos fenómenos innegables: primero, que los comediantes masculinos gozan de privilegios a la hora de producir y comercializar humor y, segundo, que esto no ha impedido que las mujeres se abran camino en este terreno hostil. A continuación, se explora cómo las mujeres crearon un tipo particular de humor: la comedia feminista.

\section{Comedia FEMinista}

Si hace décadas las mujeres comediantes vienen minando el prejuicio de que las mujeres no son graciosas, producciones como TGF redoblan la apuesta mostrando que no solo es posible ser mujer y tener sentido del humor, sino que es posible tenerlo siendo mujer y feminista. Como señala Sara Ahmed en La promesa de la felicidad (2019), las feministas no suelen ser vistas como productoras de humor, sino que, por el contrario, son acusadas de arruinar la diversión del resto. La feminista no solo no se ríe del chiste ofensivo, sino que, al no reírse, pone al descubierto el machismo del emisor y la complicidad de quienes sí se rieron. La figura de la feminista aguafiestas [feminist killjoy] desarrollada por Ahmed en ese libro captura con bastante precisión los afectos negativos asociados al feminismo y su trabajo crítico: "La palabra feminismo está saturada de infelicidad. Por el solo hecho de autoproclamarse feministas, de las feministas se piensa que vienen a destruir algo que los demás consideran no solo bueno, sino la causa de la felicidad" (2019, p. 145). Ahmed -en sintonía con las teorías de Bergson y Krefting- remarca que la risa compartida genera formas de solidaridad y que es esa solidaridad la que se ve amenazada por el feminismo. Una manera de defender el lazo social es negando que haya elementos problemáticos en lo que causa gracia, desplazando el origen del malestar a las feministas y su falta de humor. El problema, así, no sería el chiste sino el malhumor de las feministas. Ahmed invierte este orden causal, afirmando que el hecho mismo de ser consideradas el origen del malestar, minimizando o negando el componente machista del chiste, puede ser en realidad lo que ocasiona el malhumor feminista. De todas maneras, la autora hace un llamado a reapropiarnos de la figura de la feminista aguafiestas como una forma de gestar un lazo social alternativo: "Hay solidaridad en el acto de reconocernos como personas alienadas de la felicidad [...]. Puede llegar a ser una fiesta ser aguafiestas. Y debemos y podemos arruinar la fiesta" (Ahmed, 2019, p. 179).

El feminismo, al igual que otros movimientos sociales, no solo es considerado como carente de humor sino, en ocasiones, como parte de un nuevo clima de época que está destruyendo a la comedia misma. Según esta perspectiva, la victoria del discurso de lo políticamente correcto que estos movimientos fomentarían, atenta contra la esencia misma del humor: su naturaleza ofensiva. Hace unos años, este tema cobró relevancia después de que el famoso comediante Jerry Seinfeld dijera que prefiere no hacer stand-ups en universidades estadounidenses porque el público es demasiado sensible (Silman, 2015). Otros comediantes -en su mayoría varones- se hicieron eco de las palabras de Seinfeld. Chris Rock, por ejemplo, afirmó que él también dejó de hacer shows en universidades porque considera que el público es conservador, no tanto por sus creencias 
políticas sino por su miedo a ofender y quedar mal (Silman, 2015). John Cleese, por su parte, se quejó de que ya no es posible hacer chistes sobre mejicanxs pero nadie se ofende si se burla de alemanes, franceses o ingleses (Silman, 2015).

Más allá de estas críticas, el humor feminista deja en claro que el chiste, la risa y el humor no solo son compatibles con la tarea crítica, sino que, incluso, pueden convertirse en herramientas clave para burlarse del sexismo. Pero, ¿qué se entiende por humor feminista? Según Lisa Merrill, en un artículo de 1988 llamado "Feminist Humor: Rebellious and Self-Affirming", el elemento definitorio del humor feminista es que, en lugar de despreciar la experiencia de las mujeres, la reconoce y afirma. Si, tradicionalmente, se esperó que las mujeres se identificaran con comediantes que las insultaban o las tomaban como objeto de burla, la comedia feminista no es auto-denigrante, sino reivindicatoria de la posición de las mujeres. Es cierto que puede haber cuestionamientos a cómo las mujeres reproducimos roles y estereotipos socialmente impuestos, sin embargo, es un humor "auto-crítico sin ser auto-denigratorio" (Merrill, 1988, p. 277).

Uno de los puntos que Merrill defiende es que no toda mujer comediante debe ser considerada una comediante feminista. Por ejemplo, ella menciona a figuras, como Joan Rivers, ${ }^{4}$ que consiguieron abrirse camino en el mercado humorístico burlándose de otras mujeres o de ellas mismas, "de esta forma reforzando el status quo" (1988, p. 273).

De modo similar, en la introducción aPulling Our Own Strings: Feminist Humor and Satire (1980) -un libro que compila textos y viñetas cómicas hechas por mujeres-Gloria Kaufman traza una diferencia entre el humor femenino y el humor feminista. El humor femenino puede ridiculizar el sistema, pero desde una postura de aceptación ("así es la vida"). El humor feminista, en cambio, profesa "una actitud de revolución social -es decir, estamos ridiculizando un sistema social que puede y debe ser cambiado-" (Kaufman, 1980, p. 13). Mientras el humor femenino expresa un sentido de desesperanza y complacencia respecto al status quo, el humor feminista es esperanzador y revolucionario. Además, a diferencia del humor mainstream, el humor feminista no usa estereotipos de género. Por ejemplo, Kaufman sugiere que la respuesta feminista al estereotipo de la suegra no debería ser el estereotipo del suegro. Al igual que Merrill, en este libro se considera que la comedia feminista manifiesta un humor fuerte, crítico y subversivo que busca empoderar a la mujer y no denigrarla. El objeto de burla ya no son las mujeres, sino los estereotipos, los roles tradicionales que fuimos obligadas a asumir y la cultura patriarcal que da lugar a estos ideales. La ventaja de este tipo de humor, según Merril, es que la mujer que lo consume "no tiene que devaluar su propia experiencia para identificarse con una persona masculina en contra de sus intereses, tal como las mujeres deben hacer en el humor masculino tradicional" (1988, p. 279).

Si en la literatura de la década del 80 , el humor feminista estaba definido por una retórica subversiva, revolucionaria y crítica del status quo, en libros más actuales, como el editado por Fuchs Abrams, esta imagen es simultáneamente afianzada y puesta en entredicho. La autora, de hecho, se hace el siguiente interrogante: "¿puede el humor, en general, y el humor hecho por mujeres, en particular, ser visto como inherentemente subversivo o conservador?” (Fuchs Abrams, 2017, p. 8). Por un lado, ella sostiene que muchas de las contribuciones de las cómicas estadounidenses - como Amy Schumer, Sarah Silverman y Tina Fey- son efectivamente transgresoras: "estas humoristas desafían ciertas normas y presupuestos patriarcales en relación con los roles de género, la identidad y el lugar de las mujeres en la sociedad” (Fuchs Abrams, 2017, p. 1). Sin embargo, la autora también reconoce que, al ser una crítica formulada a través de la comedia, su potencial subversivo a veces se ve opacado. Evitando la romantización de la eficacia política de la comedia, Fuchs Abrams señala que "la paradoja del humor [...] es que habilita este impulso disruptivo, este desafío a la autoridad, bajo el disfraz de la aceptación social. Por eso es subversivo sin demasiados riesgos [safely subversive]" (2017, p. 8). El peligro que corre el chiste, incluso cuando busca ser transgresor, es que sea leído como meramente un chiste, como una forma de aliviar tensiones que no conduzca a una verdadera transformación de las condiciones materiales que permitieron que el chiste tenga sentido en primer lugar. 
Este artículo propone que esta dicotomía entre rebelión y aceptación, entre transgresión y conformismo, que muchas veces emerge cuando se discute el humor feminista, no es adecuada para entender el tipo de comedia que se produce en TGF. De hecho, lo que el podcast consigue es poner en cuestión la pregunta misma que Fuchs Abrams formula, a saber si la comedia de mujeres es inherentemente subversiva o conservadora. Como veremos, no hay respuesta posible para esta pregunta si se la entiende como una disyunción excluyente. Lo que apuntala el humor de TGF es el reconocimiento de que hay tanto elementos subversivos como conservadores en el modo en que llevamos adelante vidas feministas y es ese choque lo que causa gracia. Es, justamente, la incongruencia entre lo que una feminista supuestamente debe hacer y decir, y lo que efectivamente hace y dice, lo que despierta la risa y la identificación de la audiencia.

\section{El humor metafeminista de TGF}

TGF es un podcast humorístico creado en 2015 por dos cómicas: la australiana Deborah Frances-White y la danesa Sofie Hagen (quien actualmente no participa del programa). Se trata de un programa semanal, grabado frente a una audiencia en vivo, en el que participan, además de las conductoras, distintas invitadas del mundo de la comedia, especialmente anglosajón y nórdico. Además de ser un espacio donde se discute el machismo en la industria cómica, TGF es una importante plataforma para promocionar y difundir la obra de un gran número de mujeres comediantes. Se trata de un programa que pretende ser diverso en términos de sus invitadxs, reflexivo sobre el lenguaje empleado y crítico de los binarismos, la heterocisnormatividad y los privilegios raciales.

El podcast, que a la fecha lleva más de 180 episodios, aborda un tema específico cada semana, como la menstruación, la pornografía, la amistad entre mujeres, los privilegios masculinos y el orgasmo, entre otros. Los episodios siempre se inician con una serie de confesiones por parte de las conductoras que siguen la fórmula "Soy feminista, pero...". Después del "pero" se enuncian toda una serie de situaciones, en su mayoría divertidas, en las que Frances-White y Hagen (o las conductoras invitadas) se sintieron fracasar como feministas. No se trata de situaciones cómicas en sí, sino que se vuelven graciosas justamente porque le sucedieron a una feminista. Sin el "Soy feminista" que antecede al "pero", la frase sería meramente trivial o, incluso, triste. El efecto cómico, por ende, se logra a partir de la incongruencia entre las expectativas de lo que una feminista debería hacer y los permisos que ellas mismas se conceden.

Veamos algunas de estas confesiones: ${ }^{5}$

\footnotetext{
"Soy feminista, pero me bajé un podcast sobre la brecha salarial y lo escucho todas las noches porque me ayuda a dormir" (Hagen, TGF, 30/05/2016).

"Soy feminista, pero creo que soy mejor realizando sexo oral que masturbándome" (Hagen, TGF, 08/02/2016).

"Soy feminista, pero me desetiqueté de una foto tomada en una Conferencia de Mujeres en la que todas estábamos paradas frente a un cartel que decía 'Empoderadas, no asustadas' porque mis tobillos lucían gordos." (Frances-White, TGF, $22 / 01 / 2018)$.

"Soy feminista, pero cuando un comediante danés dijo que yo había llegado a la cima acostándome con otras personas, mi primer pensamiento fue: 'oh, cree que estoy en la cima'” (Hagen, TGF, 21/03/2016).

"Soy feminista, pero a veces fantaseo con ser sexualmente dominada por el famoso misógino [...] ficcional Don Draper” (Frances-White, TGF, 08/02/2016).

"Soy feminista, pero más de dos veces me afeité las piernas de emergencia en el baño de un bar porque alguien en la barra me sonrió.” (Hagen, TGF, 02/05/2016).

"Soy feminista, pero, secretamente, me gusta la película Mujer Bonita y, para ser honesta, estoy abierta a la idea de que Richard Gere pague para penetrarme en un piano de cola” (Frances-White, TGF, 04/07/2016).

"Soy feminista, pero si me dieran una libra por cada vez que me haya reído de un chiste sin gracia de un varón es probable que, finalmente, gane 26\% más que él.” (Hagen, TGF, 13/06/2016).
}

Como Frances-White reconoce, el podcast funciona como una suerte de "confesionario feminista" (TGF, 01/10/2018) en el que las comediantes, las invitadas y, por vía de la identificación, la audiencia, pueden 
ventilar las inseguridades, hipocresías y miedos que experimentan como mujeres feministas del siglo XXI. Según la conductora, esto permite "exfoliar la culpa" (TGF, 01/10/2018) para que no se transforme en vergüenza, ya que la vergüenza es una valija demasiado pesada para cargar.

Es por esto que más que hablar de humor feminista sugiero que es mejor entender TGF a partir de la idea de humor metafeminista, ya que el objeto de burla es precisamente el feminismo mismo, el modo en que lo ponemos en práctica, las incongruencias entre nuestras expectativas y nuestras acciones. La fórmula misma con la que las conductoras comienzan cada episodio "Soy feminista, pero..." apunta a las contradicciones y estándares dobles que podemos tener como feministas. El feminismo, según esta mirada, es más que una serie de principios que defendemos; se trata de una forma de vida, una ideología que se vuelve carne. Una vez que nos volvemos feministas, es imposible no leer nuestras acciones y dichos a la luz de las banderas del movimiento.

A pesar de que el feminismo es increíblemente empoderador (i.e. nos habilita a vivir vidas más creativas, menos constreñidas por los ideales impuestos, en comunidad con otras personas afines), el podcast nos recuerda que también tiene un costado hostil: puede volverse una fuente de culpa, de frustración, una vara con la cual nos juzgamos y avergonzamos. TGF nos obliga a reflexionar por qué que un movimiento de liberación puede terminar generando culpa, vergüenza y sentimientos de fracaso. ¿Qué situaciones nos hacen fracasar como feministas? ¿Qué cosas estamos dispuestas a hacer, por más que nos den culpa, y qué cosas, en cambio, nos resultan inadmisibles?

Para examinar cuáles son algunos de los permisos que se aceptan y los límites que, por el contrario, parecen infranqueables, en lo que sigue quisiera analizar las reacciones de la audiencia a las frases "Soy feminista, pero...”. Lo que el podcast permite entender es que hay ciertas violaciones a los ideales feministas que son más admitidas que otras. Son las risas, los aplausos y los silencios incómodos aquello que tomo como referencia para intentar delimitar el contorno de la comunidad feminista que se construye entre las conductoras y la audiencia.

Si bien la mayoría de las frases con las que arranca TGF suelen ser aceptadas -y por esto me refiero a que generan risas- hay algunas que son más celebradas que otras. Uno de los motivos, quizás el más obvio, es la calidad, originalidad e ingenio del comentario (de los ejemplos ya mencionados, podemos nombrar el reconocimiento de Frances-White de que su vida, a veces, no pasa el Test de Bechdel). Más allá de la calidad del chiste, hay algunos tópicos que suelen generar más empatía y aceptación. Hay dos tipos de confesiones que suelen recibir buenas respuestas: aquellas en las que el sexo, o su posibilidad, triunfa sobre los ideales feministas y aquellas en las que se manifiestan inseguridades sobre el cuerpo propio. Con respecto a lo sexual, hay toda una serie de comentarios sobre fantasear con acostarse con Don Draper, el personaje de Mad Men, que pronuncia Frances-White, que son muy celebrados. Lo mismo puede ser dicho del comentario sobre Richard Gere o la confesión de la depilación de emergencia en el baño de un bar, citados más arriba. En el primer episodio, Hagen confiesa que jamás defecó en el baño de su ex-novio para mantener la fantasía de que las mujeres somos "hadas mágicas angelicales" (TGF, 15/12/2015). En otro, la danesa se ríe del esfuerzo con el que intenta parecer sexy comiendo una banana si está frente a un muchacho atractivo (Hagen, TGF, 25/01/2015). En otro episodio, Frances-White admite que, a veces, ella quisiera ser meramente un objeto sexual. Tras las carcajadas, la comediante agrega que sería solo por una hora: "después lo quiero de vuelta, quiero el respeto" (TGF, 10/04/2017). La gran mayoría de los comentarios sobre las cosas ridículas que hacemos para seducir, el modo en que caemos en posiciones sexualmente sumisas o los permisos que nos damos con nuestros deseos sexuales suelen encontrar eco en la risa y aplauso de la audiencia.

Con respecto a las inseguridades sobre la imagen corporal, suele ser Frances-White quien pronuncia con más frecuencia este tipo de declaraciones. Al comentario sobre desetiquetarse de una foto porque sus tobillos lucían gordos que citamos previamente, podemos agregar varios chistes sobre su gordura, sobre el uso cremas anti-age y el miedo a envejecer, sobre tratamientos faciales abrasivos, etc. En un episodio, la australiana comenta que en el día de su boda secretamente quería que todos la vieran tan pero tan flaca como para creer 
que había ido demasiado lejos: "no tan lejos como para necesitar una intervención, pero sí como para que digan a mis espaldas que necesito comerme un sándwich o algo asi”" (Frances-White, TGF, 30/05/2016). En otro, confiesa que justo antes de salir al escenario, en vez de escuchar las cosas importantes que Hagen le decía, empezó a comparar su piel con la de su amiga y, viendo que la piel de la danesa lucía más joven, perfecta y bella, por un segundo la odió. Las risas que ese comentario despertaron fueron amplificadas cuando Hagen replicó: "Estás coqueteando conmigo" (TGF, 12/12/2016). Las dudas sobre el propio cuerpo, la incapacidad de aceptarse tal como una es, el deseo de verse cada vez más flacas y jóvenes, son todos tópicos que producen excelentes reacciones.

Existen otros escenarios que también son bienvenidos como, por ejemplo, el uso de estereotipos patriarcales siempre y cuando sea a conciencia y para que la comediante se salga con la suya. Cal Wilson, una conductora invitada, confesó que una vez, para salvarse de una multa de tránsito, le pidió al policía que la perdonara al grito de "soy solo una mamá" (TGF, 22/01/2018). En otro episodio, Hagen admite haberle pedido a una amiga que coqueteara con un camarero para que les dieran un descuento (TGF, 04/04/2016). También son aplaudidos los momentos en los que la frivolidad prevalece sobre lo supuestamente correcto, como cuando Frances-White reconoce haber pasado de largo un documental sobre protofeminismo en Shakespeare para ver Say Yes to the Dress, un reality show sobre mujeres que eligen vestidos de novia (TGF, 17/10/2016). En otro episodio, ella reconoce que, una vez, cuando estaba participando de una marcha por los derechos de las mujeres, entró al baño de una tienda y se quedó un rato probándose cremas faciales. Cuando salió, la marcha ya se había ido (TGF, 22/02/2016). Otro tópico similar que se repite y tiene mucho éxito es cuando la teoría feminista aburre a las comediantes. Como vimos, Hagen reconoce escuchar un podcast sobre la brecha salarial solo porque la ayuda a dormir. En otro episodio, ella le confiesa a Frances-White que ocultó un documental sobre las primeras feministas que una oyente les había regalado porque tenía miedo de que la australiana la obligara a verlo (TGF, 19/09/2016).

Sin embargo, hay ocasiones en que estos temas aparecen y no generan risa ni aplausos sino silencios incómodos, expresiones de asombro o, incluso, de lástima. También hay declaraciones que reciben reacciones mixtas: hay quienes ríen y aplauden y quienes expresan sorpresa o reprobación. A veces esas reacciones son fabricadas por las comediantes mismas, por el cambio de tono (de alegre a sombrío) o iniciando ellas mismas el gesto que la audiencia replica. Esto sucedió, por ejemplo, cuando Cal Wilson admitió en un episodio que cuando no quiere comprar algo en una tienda, dice: "Primero tengo que pedirle permiso a mi marido" (TGF, 22/01/2018). Ante esto, Frances-White se indigna y desata toda una serie de expresiones de sorpresa, repudio y algunas risas en la audiencia.

Creo que estos momentos en los que la comedia falla o produce reacciones mixtas son centrales para entender que ese nosotras-feminista que se construye en el podcast tiene sus límites. No todo es gracioso, y muchas veces lo que se pretendía gracioso termina siendo, más bien, penoso. A veces, las conductoras mismas admiten el fracaso y reconocen que su enunciado resultó ser más triste de lo que ellas habían anticipado. Cuando Hagen revela, en el primer episodio, que una vez le preguntó a un varón qué tenía que cambiar de ella para que la amara, la audiencia se quedó muda. Reconociendo lo patético de su declaración, Hagen respondió al silencio con un "sip" [yep]. En otro episodio, Frances-White admite:

Soy feminista, pero cuando estaba buscando una nueva representante y una agente de comedia me contestó un e-mail en el que la invitaba a mi show con otro e-mail que decía "no puedo considerar a ninguna mujer, puede sonar sexista pero no lo es", me metí en la bañera llorando y diciendo en voz alta "quisiera ser un hombre, quisiera ser un hombre, quisiera ser un hombre" (TGF, 22/01/2016).

En lugar de risas, la respuesta fue el silencio. Ante esto, la conductora remarcó: "Parecía más gracioso cuando lo escribí" (TGF, 22/01/2016). En otro episodio, la misma conductora relata que días antes de dar una importante charla TED en la Universidad de Cambridge, le dieron el peor corte de pelo de su vida y aunque le gustaría que la gente escuchara su conferencia, jamás la comparte porque odia cómo se ve. A continuación, añade: "No creo que un varón haga esto". Nuevamente, ante el silencio de la audiencia, la 
australiana admite: "Fue más triste de lo que pensaba" (Frances-White, TGF, 16/05/2016). El modo en que Hagen logra aliviar la tensión es remarcando su propia contradicción como feminista: "Iba a decirte que te veías bien, aunque debería haber pensado 'dijiste cosas geniales'” (TGF, 16/05/2016).

A pesar de que suele ser un tópico que genera muy buenas reacciones, la audiencia también pone límites a lo que resulta divertido en términos de las inseguridades por el propio cuerpo. En un episodio, Frances-White relata que una vez le preguntó al marido: "Si el genio del buen cuerpo saliera de la lámpara, ¿qué tres deseos le pedirías para que cambie de mí?” (TGF, 23/01/2017). La respuesta de la audiencia fue contundente: silencio y algunas expresiones de reprobación. El modo en que la conductora alivió la tensión y logró recuperar el efecto cómico fue poniendo a la audiencia en el banquillo de lxs acusadxs: "Está bien, no hace falta ser tan sentenciosxs" (TGF, 23/01/2017).

A pesar de que estos silencios pueden ser leídos como un corte en el proceso identificatorio ("hasta aquí logro empatizar"), también es posible considerar que se trata de una respuesta visceral a la sorpresa, horror o miedo de sentirse identificada con la situación penosa de la comediante. Estos afectos negativos podrían leerse como una fractura de la comunidad afectiva (un desdoblamiento entre quienes juzgan y quienes son juzgadas) o, por el contrario, como una muestra de que no solo la risa produce lazo.

Lo que los momentos de silencio, incomodidad o reacciones mixtas sí nos enseñan es que no todas las incongruencias en la vida feminista son motivo de risa. Si bien es divertido reconocer que a veces podemos ignorar nuestras banderas para tener sexo, que podemos hacer ridiculeces para vernos más jóvenes o más flacas o que podemos reproducir ideas patriarcales para salvarnos de una multa, hay otras contradicciones que dan pena, que sorprenden o que indignan.

A diferencia de programas televisivos - como las sitcoms - en los que hay una risa añadida que busca evitar el fracaso humorístico (Cheng, 2017), el hecho de que TGF esté grabado ante una audiencia en vivo, permite testear los límites de lo admitido por la audiencia por vía del humor. Querer cambiar para que un hombre te ame, llorar en el baño deseando ser un varón, o reproducir públicamente la idea de que tu marido te controla (incluso cuando no sea cierto), son ejemplos de aquello que tensiona ese nosotras-feministas. De nuevo, con esto no quiero decir que esas incongruencias sean inadmisibles en sí o que los afectos negativos no generen sentimientos de pertenencia. El punto es que, en esos instantes, el humor debe dar lugar a otro tipo de repertorio afectivo -pena, dolor, horror, sorpresa- para poder encontrar resonancia en la comunidad afectiva. TGF tiene la virtud de crear un espacio en el que podemos reírnos de, y en cierta forma aliviar, nuestras inseguridades, frustraciones y miedos. Sin embargo, su logro mayor es que no busca desentenderse del dolor y la pena que conviven con la risa. Los comentarios graciosos se alternan con las reflexiones más dolorosas y la culpa a veces sí se transforma en vergüenza. En este sentido, creo que es una virtud del programa el hacernos reír, pero más lo es el poder soportar cuando esa risa no surge, detenerse en aquellos instantes en los que la frontera entre lo trágico y lo cómico se borronea. Si bien a veces la tensión se diluye en un nuevo chiste, las conductoras no suelen desentenderse de sus fracasos humorísticos. Por el contrario, los silencios se vuelven tema, las incomodidades se remarcan, los lamentos inesperados se admiten. Se trata de un espacio simultáneamente cómico y hospitalario con los afectos tristes.

\section{5. ¿UN NUEVO HUMOR PARA UN NUEVO FEMINISMO?}

Como vimos previamente, en la bibliografía sobre el humor feminista de la década del 80 abunda la retórica del empoderamiento, la rebeldía y la crítica a los estereotipos de género. Tal como demostré en las páginas anteriores, este tipo de modelo no es del todo adecuado para entender el tipo de humor metafeminista de TFG. En el podcast, la risa surge menos de la exhibición de la fortaleza y la resistencia, que del reconocimiento de la vulnerabilidad, la imperfección y los fracasos sufridos. Así, es imposible comprender el efecto cómico del podcast si pensamos en términos maniqueos de rebeldía o conformismo. El nosotras-feministas que el programa construye no puede entenderse en el marco de una narrativa heroica 
de liberación de las restricciones del patriarcado. De hecho, parece que no hay liberación posible si por eso entendemos la posibilidad de dejar atrás los vestigios patriarcales. TGF pone en evidencia que las vidas feministas están atravesadas por elementos transgresores, pero también conservadores. Lo interesante del podcast es que esta incongruencia no es vista únicamente como algo negativo y generador de culpa, sino como objeto de burla y condición de posibilidad de la solidaridad entre pares. El proceso identificatorio no depende aquí de la idealización de la comediante, sino del reconocimiento y aceptación de una imperfección compartida. Si TGF funciona como un confesionario en el que las comediantes pueden ventilar su culpa, la risa compartida también puede ser considerada una vía a través de la cual la audiencia y las oyentes virtuales logran sentirse aliviadas. En este sentido, el podcast habilita un espacio en el que el fracaso, los miedos y la culpa no son vistos como problemas individuales, sino como afectos compartidos.

Si la caracterización de la comedia feminista de los 80 es inadecuada para entender el humor de TGF, podríamos preguntarnos si se trata del nuevo tipo de comedia que la fase actual del movimiento feminista demanda. Hay varias pensadoras que vienen reflexionando, en los últimos tiempos, sobre la relación entre el humor y la llamada cuarta ola feminista. De acuerdo con la teórica y ex-comediante, Joanne Gilbert, "como todas las formas culturales, la comedia stand-up puede ser examinada fructíferamente como un reflejo del zeitgeist actual" (2017, p. 204). Gilbert cree, de hecho, que al ser un discurso que agrada al mismo tiempo que perturba, "el humor es tanto una herramienta como un contexto para el feminismo de la cuarta ola" (2017, p. 208). Según esta perspectiva, el nuevo feminismo se caracteriza por ser pragmático, contestador y por tener sentido del humor. Además, su esfera de circulación es principalmente cibernética. La presencia virtual del feminismo contemporáneo no solo lo fortalece sino que le permite hacer uso de instrumentos digitales como los memes, los comentarios en las redes y los hashtags- para denunciar y burlarse del sexismo.

La periodista Elizabeth Held también se pregunta por el tipo de humor que las jóvenes millenials -muchas de ellas identificadas con el feminismo- consumen en la actualidad. Lo que ella sostiene es que esta nueva clase de humor, si bien puede ser anti-machista, difiere bastante de los ideales que caracterizaron a las generaciones feministas pasadas. Según la periodista, la serie Crazy Ex-Girlfriend-creada por la famosa cómica feminista Rachel Bloom y la guionista Aline Brosh McKenna- puede ser considerada el arquetipo cómico de nuestro tiempo. Esto es así ya que la serie deja en claro que, más que sacrificarse por una causa, lo importante es seguir nuestro corazón (Held, 2016). En Crazy Ex-Girlfriend, la protagonista principal, Rebecca Bunch, abandona una importante carrera como abogada en Nueva York para mudarse a West Covina, una pequeña ciudad de California donde vive su ex novio, a quien pretende reconquistar. La decisión de Rebecca podría ser leída según la fórmula de TGF: "Soy feminista pero prefiero dejar mi trabajo exitoso para ir en busca del amor". La protagonista privilegia la felicidad sobre el éxito y es esto lo que, según Held, resuena entre las millennials. Es por esto, según la periodista, que las feministas de la segunda ola están tan decepcionadas con las jóvenes de hoy, a saber porque "han abandonado todo por lo que ellas lucharon" (Held, 2016). Para las feministas de la generación previa, "las mujeres tienen un deber con la causa feminista", aunque esta vaya en contra de sus intereses individuales (Held, 2016). Abandonar el camino del éxito para conquistar a un ex no sería admisible para el nosotras-feministas previo, pero sí para las feministas de hoy.

¿Podemos leer el humor metafeminista de TGF en el marco de estas reflexiones? Si bien TGF pone en primer plano los compromisos que una feminista contemporánea está dispuesta a hacer, analizarlo en el marco de un quiebre generacional, al estilo de Held, es confuso. En primer lugar, porque implicaría que las feministas previas no tuvieron dificultades a la hora de conciliar sus ideales con sus deseos personales. ¿Podemos realmente afirmar que la incongruencia entre el deber ser y el ser es un problema actual que las feministas previas no tuvieron? En segundo lugar, porque lo que las comediantes de TGF permiten entender es que no se trata, nuevamente, de sacrificarse por el feminismo o renunciar a él. Vivir una vida feminista no implica estar constantemente transgrediendo lo heredado, rompiendo estructuras, subvirtiendo normas sexistas. El punto del podcast es aprender a reconocer las propias contradicciones e intentar no ser tan duras cuando juzgamos a otras y a nosotras mismas. 
En lugar de pensar que hay una forma de humor que se corresponde con una época determinada, creo que es más útil preguntarnos qué herramientas nos ofrece cierto producto cultural para reflexionar sobre los problemas de su tiempo. En este sentido, me gustaría finalizar este artículo resaltando algunos recursos que TGF nos brinda para repensar un tópico recurrente cuando se habla de la cuarta ola feminista: las guerras de frontera que se construyen tras la masificación del feminismo, es decir, quiénes son las feministas reales y quiénes las que siguen una moda.

Una de las notas asociadas a esta nueva fase del feminismo es su marcada circulación a través de las redes sociales y otras plataformas virtuales. El costado positivo del ciber-activismo es que permite llegar a muchísimas personas, probablemente más que otras formas de propagación. Sin embargo, también debe enfrentarse a los problemas usuales de las redes sociales como la presencia de trolls, el uso agresivo de comentarios y la impunidad que conlleva el anonimato de algunos perfiles, entre otros. Con respecto a esto último, hace algunos años la periodista de The Huffington Post, Jennifer Simpkins, publicó una nota de denuncia sobre nuevas formas de exclusión y violencia al interior del ciber-feminismo. En la nota, ella sostenía que el feminismo de la cuarta ola corre el riesgo de convertirse en una nueva versión de la película Mean Girls. Recordemos que en ese filme cómico una adolescente, interpretada por Lindsay Lohan, es víctima de bullying por parte de las chicas populares de la nueva escuela a la que asiste. Simpkins afirma que, en varias ocasiones, ella se sintió igualmente atacada, aunque de forma virtual, por colegas feministas que cuestionaban los posteos, videos o notas que ella subía a sus redes sociales. Simpkins teme que, a pesar de ser un movimiento que reivindica la sororidad, el nuevo feminismo virtual termine reproduciendo gestos violentos contra otras mujeres: “¿Por qué algunas ciber-feministas creen honestamente que sus opiniones existen en un plano intelectual superior al del resto de las mujeres? ¿Qué las lleva a menospreciar a otras mujeres, a veces salvajemente, en foros públicos?" (Simpkins, 2014).

Además de su propagación virtual, un segundo elemento que suele estar vinculado a la cuarta ola del feminismo es su creciente popularización, es decir, el hecho de que un gran número de personas -incluyendo actrices, cantantes, celebridades, personalidades mediáticas- actualmente se identifican con el movimiento. Esto sucede tanto en el mundo anglosajón como en nuestra región. En Argentina, por ejemplo, en los últimos años hubo una participación masiva de la ciudadanía en movilizaciones como el $\mathrm{Ni}$ una menos o el Paro de mujeres. Esto coincide con un importante interés y cobertura mediática de temas de la agenda feminista como los femicidios, la violencia sexista y la lucha por la legalización del aborto. Si bien en este artículo no se examinó la tradición humorística argentina, creo que es posible extrapolar algunas de las discusiones sobre la comunidad afectiva de TGF para revisar las reacciones mixtas que se produjeron en nuestro país tras la popularización del feminismo. ¿A qué me refiero con reacciones mixtas? Al hecho de que hay voces que celebran las posibilidades que se abren cuando el feminismo se vuelve más popular y otras que lamentan las consecuencias nocivas de la masificación (como por ejemplo el uso estratégico que algunxs políticxs y periodistas hacen del movimiento para subir su rating). A principios del 2018, la investigadora argentina, Carolina Spataro, publicó una nota en la revista Bordes que pone sobre el tapete algunas de las dificultades y contradicciones que se siguen de la popularización del feminismo y de la incorporación de sus temas a los medios masivos. Spataro analiza las reacciones de algunas feministas cuando ciertos personajes mediáticos, como Araceli González, Wanda Nara o Jorge Rial, se acercan al movimiento. La nota se titula "Abajo el feministómetro" y anticipa la idea central del texto: tenemos que dejar de medir las acciones feministas con una vara universal, imposible de cumplir. En palabras de la autora: "en un momento de expansión del feminismo, ¿qué sentido tiene elaborar un check list que deberían cumplir las mujeres para otorgarles la credencial de feminista?" (Spataro, 2018, p. 28). Al igual que Simpkins, la autora se preocupa por los procesos de legitimación al interior del feminismo que llevan a que ciertas voces expertas se crean autorizadas a demarcar entre las feministas verdaderas y las meras impostoras (i.e. las que siguen una moda, las que lucran con el cuerpo, las que alguna vez reprodujeron frases o prácticas machistas). En lugar de levantar nuevos 
muros entre buenas y malas feministas, Spataro demanda: "erradiquemos la policía del feminismo de una buena vez" (2018, p. 28).

En el marco de estos señalamientos, creo que TGF puede brindarnos herramientas para repensar algunas de estas preocupaciones. En especial, creo que tiene algo para decir sobre cómo manejar el sentimiento de "fracaso" que podemos tener como feministas y la culpa que nos genera no ser una feminista ideal. Como vimos, el podcast nos recuerda que ser feminista conlleva un cambio sustancial en nuestra forma de ser en el mundo. El feminismo es más que un conjunto de creencias a las que podemos adherir, es un modo de vivir la vida. Una vez que nos reconocemos parte del movimiento, es difícil no leer nuestras acciones, nuestros dichos, hasta nuestras fantasías y nuestros sueños, en términos de su congruencia (o no) con las banderas que levantamos. Quienes nos consideramos feministas, lo valoramos ya que nos permite vivir vidas más libres y creativas. No obstante, el podcast nos recuerda que tiene también un costado adverso: puede volverse una nueva norma que restringe y lastima, una vara con la que juzgamos, duramente, acciones propias y ajenas. En este sentido, TGF nos obliga a preguntarnos: ¿Hay feministas buenas y feministas malas? ¿Cómo las diferenciamos? ¿No serán estas demarcaciones vestigios patriarcales al interior de un movimiento antipatriarcal?

Aunque coincido con el pedido de Spataro de erradicar la policía del feminismo -y esto vale, incluso, para una misma- creo que el podcast deja en claro que es muy difícil volverse feminista y no estar constantemente revisando cómo una conduce su vida, cómo podría conducirla mejor, cuáles son las incongruencias entre lo que hacemos y lo que pensamos. TGF revela al feminismo como un movimiento profundamente autoreflexivo, incluso al punto de hacernos revisar hasta el más mínimo detalle de nuestra vida cotidiana. En Vivir una vida feminista (2018), Ahmed describe con bastante precisión esta sensación omnipresente y abarcativa del feminismo que TGF destaca:

La conciencia feminista puede ser como un interruptor que está encendido. Apagarlo podría ser necesario para sobrevivir en el mundo en el que vivimos, que no es un mundo feminista. La consciencia feminista es cuando el botón de encendido es la posición por defecto. A menos que lo apagues, estás encendida. [...] No hay duda: puede ser agotador. (Ahmed, 2018, p. 54).

Agotamiento, culpa, vergüenza, ansiedad, frustración. Todo eso puede generar el feminismo. Pero también alegría, risa, comunidad, empatía, identificación. Las feministas podemos ser transgresoras, subversivas y radicales. Pero también reproducir ideas conservadoras, fallar, elegir las batallas, hacer lo que podemos y lo que nos da el cuerpo. TGF no hace un llamado a dejar de juzgar, a otras y a nosotras mismas, como si eso fuera posible. Pero sí a reírnos de nuestras actitudes policiales y, con esa risa, quitarle un poco de fuerza y solemnidad a los ideales regulativos que nos vulneran y sofocan. El podcast es una invitación a ser más amables con nosotras y con las otras, a entender que no es posible ser una feminista perfecta, a aprender a vivir con las propias contradicciones y a intentar comprender las contradicciones de otras. La comunidad afectiva que se despliega en el podcast no obliga a expulsar los sentimientos negativos ni a esconder las disonancias entre sus miembros. La culpa, la vergüenza y la frustración no tienen por qué aislarnos. Son parte de la infraestructura emocional de una comunidad sostenida en el reconocimiento de una vulnerabilidad en común.

Habrá ocasiones en las que haremos cosas que nos den culpa y podremos reprocharnos o intentar cambiar. Pero también habrá momentos en los que el fracaso nos ayude a entender que no somos perfectas y que no es tan fácil desandar los caminos aprendidos. En esos instantes, nuestras incongruencias, en lugar de dar vergüenza, pueden darnos gracia. El podcast no nos incita a dejar de ser críticas ni a abrazar el relativismo. Se trata, más bien, de una invitación a ser más empáticas, más comprensivas, más consideradas con nosotras y con las otras. Si logramos esto, podremos comenzar a construir comunidades basadas menos en requisitos absolutos que en la admisión de las imperfecciones, contradicciones y fracasos que todas experimentamos. TGF crea un espacio para que esa comunidad puede reírse de sus fallas y reconocer sus miserias, un espacio en el que se puede celebrar el feminismo sin negar su costado hostil. Quizás sea una comunidad con culpa, 
vulnerable y fallida pero también alegre, empática y hospitalaria. Y, sobre todas las cosas, con sentido del humor.

\section{ReFERENCIAS}

Ahmed, S. (2018). Vivir una vida feminista. Barcelona: Bellaterra.

Ahmed, S. (2019). La promesa de la felicidad. Una crítica cultural al imperativo de la alegría. Buenos Aires: Caja Negra. Berlant, L. y Ngai, S. (2017). Comedy has Issues. Critical Inquiry, 43(2) 233 -249.

Bergson, H. (1962). La risa. Ensayo sobre la significación de lo cómico. Buenos Aires: Losada.

Cheng, W. (2017). Taking Back the Laugh: Comedic Alibis, Funny Fails. Critical Inquiry, 43(2), 528-549.

Freud, S. (1991 [1905]). El chiste y su relación con el inconsciente. En Obras completas. Vol. 8. Buenos Aires: Amorrortu Ediciones.

Fuchs Abrams, S. (2017). No Joke: Transgressive Humor of American Women Writers. En Fuchs Abrams (ed.) Transgressive Humor of American Women Writers (pp.1-16). Cham: Palgrave Macmillan.

Gilbert, J. (2017). 'My Mom's a Cunt': New Bawds Ride the Fourth Wave. En Fuchs Abrams (ed.) Transgressive Humor of American Women Writers (pp. 2013-230). Cham: Palgrave Macmillan.

Held, E. (18 de abril de 2016). Millennials Prefer The 'Crazy Ex-Girlfriend' Career Path Over Feminism. The Federalist. Recuperado de: http://thefederalist.com/2016/04/18/millennials-prefer-the-crazy-ex-girlfriend-career-path-o ver-feminism/

Hitchens, C. (enero de 2007). Why Women Aren't Funny? Vanity Fair. Recuperado de: https://www.vanityfair.co $\mathrm{m} /$ culture/2007/01/hitchens 200701

Hobbes, T. (1998). Leviathan. Oxford: Oxford University Press.

Kaufman, G. (1980). Introduction. En Kaufman y Blakely (ed.). Pulling Our Own Strings: Feminist Humor and Satire (pp.13-16). Bloomington: Indian University Press.

Kein, K. (2015). Review: Recovering Our Sense of Humor: New Directions in Feminist Humor Studies. Feminist Studies, 41(3), 671-681.

Krefting, R. (2014). All Joking Aside: American Humor and its Discontents. Baltimore: Johns Hopkins University Press.

Merrill, L. (1988). Feminist humor: Rebellious and self - affirming. Women's Studies: An inter-disciplinary journal, $15(1-3), 271-280$.

Silman, A. (11 de junio de 2015). 10 famous comedians on how political correctness is killing comedy: "We are addicted to the rush of being offended". Salon. Recuperado de: https://www.salon.com/2015/06/10/10_famous_comedians_on_how_political_correctness_is_killing_c omedy_we_are_addicted_to_the_rush_of_being_offended/

Simpkins, J. (19 de enero de 2014). 'You Can't Sit With Us!' - How Fourth-Wave Feminism Became 'Mean Girls'. HuffPost Blog. Recuperado de: https://www.huffingtonpost.co.uk/jennifer-simpkins/feminism-fourth-wave-b ecame-mean-girls_b_4616597.html

Spataro, C. (2018). Abajo el Feministómetro. Bordes. Revista de política, derecho y sociedad, número 8, 19-28. Recuperado de: http://revistabordes.com.ar/wp-content/uploads/2018/08/2_Abajo_feministometro.pdf

Zink, V. (2019). Affective communities. En Jan Slaby y Christian von Scheve (ed.). Affective Societies (pp. 289-299). Nueva York: Routledge.

\section{Episodios citados de The Guilty Feminist}

Frances-White, D. y Hagen, S. (Presentadoras). (15 de diciembre de 2015). Nudity with Shappi Khorsandi [Audio en podcast]. Recuperado de https://guiltyfeminist.com/episode/?episode $=1$ 
Frances-White, D. y Hagen, S. (Presentadoras). (25 de enero de 2016). Food with Emma Kennedy [Audio en podcast]. Recuperado de https://guiltyfeminist.com/episode/?episode $=4$

Frances-White, D. y Hagen, S. (Presentadoras). (08 de febrero de 2016). Apologising with Jo Caulfield [Audio en podcast]. Recuperado de https://guiltyfeminist.com/episode/?episode $=6$

Frances-White, D. y Hagen, S. (Presentadoras). (22 de febrero de 2016). Porn with Sara Pascoe [Audio en podcast]. Recuperado de https://guiltyfeminist.com/episode/?episode $=8$

Frances-White, D. y Hagen, S. (Presentadoras). (21 de marzo de 2016). Exercise with Jessamyn Stanley [Audio en podcast]. Recuperado de https://guiltyfeminist.com/episode/?episode $=13$

Frances-White, D. y Hagen, S. (Presentadoras). (04 de abril de 2016). Women's Magazines with Sarah Millican [Audio en podcast]. Recuperado de https://guiltyfeminist.com/episode/?episode $=15$

Frances-White, D. y Hagen, S. (Presentadoras). (18 de abril de 2016). Dating with Roisin Conaty [Audio en podcast]. Recuperado de https://guiltyfeminist.com/episode?episode $=17$

Frances-White, D. y Hagen, S. (Presentadoras). (02 de mayo de 2016). Representation with Margaret Cabourn-Smith [Audio en podcast]. Recuperado de https://guiltyfeminist.com/episode/ ?episode $=19$

Frances-White, D. y Hagen, S. (Presentadoras). (16 de mayo de 2016). Crazy Cat Lady with Celia Pacquola [Audio en podcast]. Recuperado de https://guiltyfeminist.com/episode/?episode $=21$

Frances-White, D. y Hagen, S. (Presentadoras). (30 de mayo de 2016). Femininity with Hannah Gadsby [Audio en podcast]. Recuperado de https://guiltyfeminist.com/episode/?episode $=24$

Frances-White, D. y Hagen, S. (Presentadoras). (13 de junio de 2016). Worth with Sarah Millican [Audio en podcast]. Recuperado de https://guiltyfeminist.com/episode/?episode $=26$

Frances-White, D. y Hagen, S. (Presentadoras). (04 de julio de 2016). Anger with Lolly Adefope [Audio en podcast]. Recuperado de https://guiltyfeminist.com/episode/?episode $=29$

Frances-White, D. y Hagen, S. (Presentadoras). (19 de septiembre de 2016). Shoes with Jessica Fostekew [Audio en podcast]. Recuperado de https://guiltyfeminist.com/episode/?episode $=40$

Frances-White, D. y Hagen, S. (Presentadoras). (17 de octubre de 2016). Promiscuity with Sanne Søndergaard [Audio en podcast]. Recuperado de https://guiltyfeminist.com/episode/?episode $=44$

Frances-White, D. y Hagen, S. (Presentadoras). (12 de diciembre de 2016). Being Bossy with Carrie Quinlan [Audio en podcast]. Recuperado de https://guiltyfeminist.com/episode/?episode $=54$

Frances-White, D. (Presentadora). (10 de abril de 2017). Democracy with Jess Phillips MP [Audio en podcast]. Recuperado de https://guiltyfeminist.com/episode/?episode $=72$

Frances-White, D. (Presentadora). (22 de enero de 2018). Hope with Hannah Gadsby [Audio en podcast]. Recuperado de https://guiltyfeminist.com/episode?episode $=114$

Frances-White, D. (Presentadora). (01 de octubre de 2018). Taking Control of the Narrative with Susan Wokoma and special guests Ella Hickson, Samuel West and Lara Rossi [Audio en podcast]. Recuperado de https://guilt yfeminist.com/episode/?episode $=151$

\section{Notas}

1 El podcast The Guilty Feminist se inicia en diciembre de 2015 y sigue vigente. Fue creado por la australiana Deborah Frances-White y la danesa Sofie Hagen. Es grabado en vivo en distintos escenarios internacionales (Europa, EEUU, Australia, entre otros). A fines de 2019 el podcast tiene 180 episodios y cuenta con más de 60 millones de descargas. Para escuchar los episodios completos o tener más información, ver la página web: http://www.guiltyfeminist.com

2 Todas las citas del inglés al español fueron realizadas por la autora de este artículo.

3 Dado que el podcast que examino es producido en inglés y por comediantes europeas, en este artículo me centraré en el mundo humorístico anglosajón.

4 Joan Rivers (1933-2014) era una comediante, conductora y productora estadounidenses conocida por su humor controversial, agudo y despreciativo hacia otras celebridades (y hacia sí misma). Fue conductora del famoso show Fashion Police en E! 
5 La mayoría de las frases que analizo en este apartado son del año 2016 cuando el podcast todavía estaba conducido por la dupla Frances-White/Hagen. Cuando Hagen abandona el programa a principios del 2017, distintas invitadas ejercieron el rol de co-conductoras. Creo que, al estar en manos de las mismas dos conductoras, los episodios del 2016 permiten identificar más claramente ciertos patrones y regularidades en las confesiones. De todas formas, incluyo algunas declaraciones posteriores, especialmente del 2018, para mostrar la continuidad de temas recurrentes. 